\title{
AB INITIO CALCULATIONS OF THE OPTICAL AND MAGNETO-OPTICAL PROPERTIES OF MODERATELY CORRELATED SYSTEMS: ACCOUNTING FOR CORRELATION EFFECTS
}

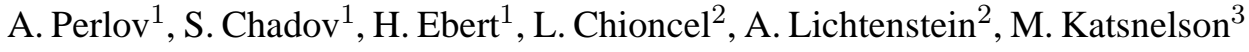 \\ ${ }^{1}$ University of Munich, Butenandstrasse 5-13, D-81377, Munich, Germany \\ ${ }^{2}$ University of Nijmegen, NL-6526 ED Nijmegen, The Netherlands \\ ${ }^{3}$ Uppsala University, P.O.Box 530, S-751 21 Uppsala, Sweden
}

\begin{abstract}
The influence of dynamical correlation effects on the magneto-optical properties of ferromagnetic $\mathrm{Fe}$ and $\mathrm{Ni}$ has been investigated. In addition the temperature dependence of the self-energy and its influence on the DOS and optical conductivity is considered. Magneto-optical properties were calculated on the basis of the one-particle Green's function, which was obtained from the DMFT-SPTF procedure. It is shown that dynamical correlations play a rather important role in weakly correlated $\mathrm{Fe}$ and substantially change the spectra for moderately correlated Ni. Magneto-optical properties obtained for both systems are found in better agreement with experiment than by conventional LDA calculations.
\end{abstract}

\section{Introduction}

Much information on the electronic structure of magnetic solids is gained by optical and magneto-optical measurements, being useful tools for analyzing the dispersion of (quasi-particle) bands. However, measured optical and magneto-optical spectra can hardly be interpreted without accompanying theoretical calculations. For this purpose one in general has to solve a corresponding many-electron problem, which is impossible without the use of more or less severe approximations. For materials where the kinetic energy of the electrons is more important than the Coulomb interactions, the most successful first principles method is the Local (Spin-) Density Approximation (L(S)DA) to the Density Functional theory (DFT) [1], where the many-body problem is mapped onto a non-interacting system with a one-electron exchange-correlation potential approximated by that of the homogeneous electron gas. For the last two 
decades $a b$ initio calculations of the optical and magneto-optical properties of solids based on this approximation yielded a good basis for such an interpretation, often leading to a quantitative agreement between theoretical and experimental spectra. The situation is very different when we consider more strongly correlated materials, (systems containing $\mathrm{f}$ and $\mathrm{d}$ electrons) since in all the calculations the LDA eigen-energies are implicitly interpreted to be the oneparticle excitation energies of the system. It is well known that there are two possible sources of error connected with that approach: Firstly, the LDA provides only an approximate expression for the (local) exchange-correlation potential. Secondly, even with the exact exchange-correlation potential at hand, one is left with the problem that there is no known correspondence between the Kohn-Sham eigen-energies and the one-particle excitation energies [2-5].

For an exact description of the excitation energies the non-local self-energy has to be considered. This, however, constitutes a many-body problem. Therefore, DFT-LDA calculations must be supplemented by many-body methods to arrive at a realistic description of the one-particle excitations in correlated systems. To give an example, let us mention the GW approximation [6] which is well suited for the case of insulators and semi-conductors and has also been applied successfully to transition metals [6-9]. Another approach is to consider the Hubbard-type models where those Coulomb-interaction terms are included explicitly that are assumed to be treated insufficiently within DFTLDA. Already the simplest Hartree-Fock like realization of such an approach called LDA+U [10] scheme allowed to improve considerably the description of the optical and magneto-optical spectra of strongly correlated systems (mostly containing rare earths elements $[11,12]$ ). The main advantage of the LDA+U scheme is the energy independence of the self-energy which allows to use only slightly modified standard band structure methods for calculating optical and magneto-optical spectra. On the other hand the scheme works rather good only for extremely correlated systems, where Coulomb interactions (U) prevail considerably over the kinetic energy (bandwidth W). For moderately correlated systems $(\mathrm{U} \approx \mathrm{W})$ which applies for most $3 d$ and $5 f$ elements and their compounds one has to take into account a non-Hermitian energy dependent self-energy to get a reasonable description of the electronic structure. Nowadays there are several approaches available to deal with this situation. The most advanced one is the Dynamical Mean-Field Theory (DMFT) [13]. DMFT is a successful approach to investigate strongly correlated systems with local Coulomb interactions. It uses the band structure results calculated, for example, within LDA approximation, as input and then missing electronic correlations are introduced by mapping the lattice problem onto an effective single-site problem which is equivalent to an Anderson impurity model [14]. Due to this equivalence a variety of approximative techniques have been used to solve the DMFT equations, such as Iterated Perturbation Theory (IPT) 
[13, 15], Non-Crossing Approximation (NCA) [16, 17], numerical techniques like Quantum Monte Carlo simulations (QMC) [18], Exact Diagonalization (ED) [15, 19], Numerical Renormalization Group (NRG) [20], or Fluctuation Exchange (FLEX) [21-23]. The DMFT maps lattice models onto quantum impurity models subject to a self-consistency condition in such a way that the many-body problem for the crystal splits into a single-particle impurity problem and a many-body problem of an effective atom. In fact, the DMFT, due to numerical and analytical techniques developed to solve the effective impurity problem [13], is a very efficient and extensively used approximation for energy-dependent self energy $\Sigma(\omega)$. At present LDA+DMFT is the only available ab initio computational technique which is able to treat correlated electronic systems close to a Mott-Hubbard MIT (Metal-Insulator Transition), heavy fermions and $f$-electron systems.

Concerning the calculation of the optical spectra we have to face the following problem: one particle wave functions are not defined any more and the formalism has to applied in the Green function representation. Such a representation has already been derived [24] and successfully applied for calculations in the framework of Korringa-Kohn-Rostoker (KKR) Green-function method for LSDA calculations. The only drawback of such an approach is that it is highly demanding as to both computational resources and computational time.

In this paper we propose a simplified way to calculate optical and magnetooptical properties of solids in the Green function representation based on variational methods of band structure calculations.

The paper is organized as following: in section 2 the formalism for Green's function calculations of optical and magneto-optical properties that account for many-body effects through an effective self-energy is presented. Then, the DMFT-SPTF method for the calculation of the self-energy is considered. In section 3 the obtained results of our calculations for $\mathrm{Fe}$ and $\mathrm{Ni}$ are discussed and compared with experimental ones. The last section 4 contains the conclusion and an outlook.

\section{Green's function calculations of the conductivity tensor}

Optical properties of solids are conventionally described in terms of either the dielectric function or the optical conductivity tensor which are connected via the simple relationship:

$$
\sigma_{\alpha \beta}(\omega)=-\frac{i \omega}{4 \pi}\left(\varepsilon_{\alpha \beta}(\omega)-\delta_{\alpha \beta}\right)
$$

The optical conductivity is connected directly to the other optical properties. For example, the Kerr rotation $\theta_{K}(\omega)$ and so-called Kerr ellipticity $\varepsilon_{K}(\omega)$ for 
small angles and $\left|\varepsilon_{x y}\right| \ll\left|\varepsilon_{x x}\right|$ can be calculated using the expression [25]:

$$
\theta_{K}(\omega)+i \varepsilon_{K}(\omega)=\frac{-\sigma_{x y}(\omega)}{\sigma_{x x}(\omega)\left[1+\frac{4 \pi}{\omega} \sigma_{x x}(\omega)\right]^{1 / 2}} .
$$

The reflectivity coefficient $r$ is given by

$$
r=\frac{(n-1)^{2}+k^{2}}{(n+1)^{2}+k^{2}}
$$

with $n$ and $k$ being the components of the complex refractive index, namely refractive and absorptive indices, respectively. They are connected to the dielectric function via:

$$
n+i k=\left(\varepsilon_{x x}+i \varepsilon_{x y}\right)^{1 / 2} .
$$

Microscopic calculations of the optical conductivity tensor are based on the Kubo linear response formalism [26]:

$$
\sigma_{\alpha \beta}(\omega)=-\frac{1}{\hbar \omega V} \int_{-\infty}^{0} d \tau e^{-i(\omega+i \eta) \tau}\left\langle\left[J_{\beta}(\tau), J_{\alpha}(0)\right]\right\rangle
$$

involving the expectation value of the correlator of the electric current operator $J_{\alpha}(\tau)$. In the framework of the quasiparticle description of the excitation spectra of solids the formula can be rewritten in the spirit of the Greenwood approach and making use of the one-particle Green function $G(E)$ :

$$
\begin{array}{r}
\sigma_{\alpha \beta}(\omega)=\frac{i \hbar}{\pi^{2} V} \int_{-\infty}^{\infty} d E \int_{-\infty}^{\infty} d E^{\prime} f(E-\mu) f\left(\mu-E^{\prime}\right) \\
{\left[\frac{\operatorname{Tr}\left\{\hat{j}_{\alpha} \Im G\left(E^{\prime}\right) \hat{j}_{\beta} \Im G(E)\right\}}{\left(E^{\prime}-E+i \eta\right)\left(\hbar \omega+E-E^{\prime}+i \eta\right)}+\right.} \\
\\
\left.\frac{\operatorname{Tr}\left\{\hat{j}_{\beta} \Im G\left(E^{\prime}\right) \hat{j}_{\alpha} \Im G(E)\right\}}{\left(E^{\prime}-E+i \eta\right)\left(\hbar \omega+E^{\prime}-E+i \eta\right)}\right],
\end{array}
$$

where $\Im G(E)$ stands for the anti-Hermitian part of the Green's function, $f(E)$ is the Fermi function and $V$ is the volume of a sample. Taking the zero temperature limit and making use of the analytical properties of the Green's function one can get a simpler expression for the absorptive (anti-Hermitian) part of the conductivity tensor:

$$
\sigma_{\alpha \beta}^{(1)}(\omega)=\frac{1}{\pi \omega} \int_{E_{F}-\omega}^{E_{F}} d E \operatorname{tr}\left[\hat{j}_{\alpha} \Im G(E) \hat{j}_{\beta} \Im G(E+\hbar \omega)\right] .
$$


The dispersive part of $\sigma_{\alpha \beta}(\omega)$ is connected to the absorptive one via a Kramers-Kronig relationship.

The central quantity entering expression Eq.(7) is the one-particle Green's function defined as a solution of the equation:

$$
\left[\hat{H}_{0}+\hat{\Sigma}(E)-E\right] \hat{G}(E)=\hat{I},
$$

where $\hat{H}_{0}$ is a one-particle Hamiltonian including the kinetic energy, the electronion Coulomb interaction and the Hartree potential, while the self-energy $\hat{\Sigma}(E)$ describes all static and dynamic effects of electron-electron exchange and correlations. The L(S)DA introduces the self-energy as a local, energy independent exchange-correlation potential $V_{x c}(r)$. As the introduction of such an additional potential does not change the properties of $\hat{H}_{0}$ we will incorporate this potential to $\hat{H}_{L D A}$ and subtract this term from the self-energy operator. This means that the self energy $\Sigma$ used in the following is meant to describe exchange and correlation effects not accounted for within LSDA.

With a choice of the complete basis set $\{|i\rangle\}$ the Green's function can be represented as:

$$
G(E)=\sum_{i j}|i\rangle G_{i j}(E)\langle j|,
$$

with the Green's matrix $G_{i j}$ being defined as

$$
G_{i j}(E)=[\langle i|\hat{H}| j\rangle-E\langle i \mid j\rangle+\langle i|\hat{\Sigma}(E)| j\rangle]^{-1} .
$$

Dealing with crystals one can make use of Bloch's theorem when choosing basic functions $\left|i_{\mathbf{k}}\right\rangle$. This leads to the k-dependent Green's function matrix

$$
G_{i j}^{\mathbf{k}}(E)=\left[H_{i j}^{\mathbf{k}}-E O_{i j}^{\mathbf{k}}+\Sigma_{i j}^{\mathbf{k}}(E)\right]^{-1} .
$$

Introducing the anti-Hermitian part of the Green's function matrix as

$$
\mathcal{G}_{i j}^{\mathbf{k}}(E)=\frac{i}{2}\left[G_{i j}^{\mathbf{k}}(E)-G_{j i}^{\mathbf{k}}(E)\right]
$$

and taking into account the above mentioned translational symmetry we obtain the following expression for the absorptive part of the optical conductivity:

$$
\sigma_{\alpha_{\beta}}^{a b s}=\frac{1}{\pi \omega} \int_{E_{F}-\hbar \omega}^{E_{F}} d E \int d^{3} k \sum_{i j} \mathcal{J}_{i j}^{\alpha}(\mathbf{k}, E) \mathcal{J}_{j i}^{\beta}(\mathbf{k}, E+\hbar \omega)
$$

with

$$
\mathcal{J}_{i j}^{\alpha}(\mathbf{k}, E)=\sum_{n} \mathcal{G}_{i n}^{\mathbf{k}}(E)\left\langle n_{\mathbf{k}}\left|\hat{j}^{\alpha}\right| j_{\mathbf{k}}\right\rangle
$$


The efficiency and accuracy of the approach is determined by the choice of $\left|i_{\mathbf{k}}\right\rangle$. One of the computationally most efficient variational methods is the Linear Muffin-Tin Orbitals method [27] which allows one to get a rather accurate description of the valence/conduction band in the range of about $1 \mathrm{Ry}$, which is enough for the calculations of the optical spectra $(\hbar \omega<6-8 \mathrm{eV})$. This method has been used in the present work. A detailed description of the application of the above sketched approach in the framework of LMTO can be found elsewhere [28].

\section{Calculation of the self-energy}

The key point for accounting of many-body correlations in the present approach is the choice of approximation for the self-energy. As it was discussed in the Introduction one of the most elaborated modern approximation is DMFT.

For the present work we have chosen one of the most computationally efficient variants of DMFT: Spin polarized $T$-matrix plus fluctuation exchange (SPTF) approximation [23], which is based on the general many-body Hamiltonian in the $\mathrm{LDA}+\mathrm{U}$ scheme:

$$
\begin{aligned}
H & =H_{t}+H_{U} \\
H_{t} & =\sum_{\lambda \lambda^{\prime} \sigma} t_{\lambda \lambda^{\prime}} c_{\lambda \sigma}^{+} c_{\lambda^{\prime} \sigma} \\
H_{U} & =\frac{1}{2} \sum_{\left\{\lambda_{i}\right\} \sigma \sigma^{\prime}}\left\langle\lambda_{1} \lambda_{2}|v| \lambda_{1}^{\prime} \lambda_{2}^{\prime}\right\rangle c_{\lambda_{1} \sigma}^{+} c_{\lambda_{2} \sigma^{\prime}}^{+} c_{\lambda_{2}^{\prime} \sigma^{\prime}} c_{\lambda_{1}^{\prime} \sigma},
\end{aligned}
$$

where $\lambda=i m$ are the site number $(i)$ and orbital $(m)$ quantum numbers, $\sigma=\uparrow, \downarrow$ is the spin projection, $c^{+}, c$ are the Fermion creation and annihilation operators, $H_{t}$ is the effective single-particle Hamiltonian from the LDA, corrected for the double-counting of average interactions among correlated electrons as it will be described below. The matrix elements of the screened Coulomb potential are defined in the standard way

$$
\langle 12|v| 34\rangle=\int d \mathbf{r} d \mathbf{r}^{\prime} \psi_{1}^{*}(\mathbf{r}) \psi_{2}^{*}\left(\mathbf{r}^{\prime}\right) v\left(\mathbf{r}-\mathbf{r}^{\prime}\right) \psi_{3}(\mathbf{r}) \psi_{4}\left(\mathbf{r}^{\prime}\right),
$$

where we define for briefness $\lambda_{1} \equiv 1$ etc. A general SPTF scheme has been presented recently [23]. For $d$ electrons in cubic structures where the onesite Green function is diagonal in orbital indices the general formalism can be simplified. First, the basic equation for the $T$-matrix which replaces the effective potential in the SPTF approach reads

$$
\begin{array}{r}
\left\langle 13\left|T^{\sigma \sigma^{\prime}}(i \Omega)\right| 24\right\rangle=\langle 13|v| 24\rangle \frac{1}{\beta} \sum_{\omega} \sum_{56}\langle 13|v| 56\rangle \times \\
G_{5}^{\sigma}(i \omega) G_{6}^{\sigma^{\prime}}(i \Omega-i \omega)\left\langle 56\left|T^{\sigma \sigma^{\prime}}(i \Omega)\right| 24\right\rangle,
\end{array}
$$


where $\omega=(2 n+1) \pi T$ are the Matsubara frequencies for temperature $T \equiv$ $\beta^{-1}(n=0, \pm 1, \ldots)$.

At first, we should take into account the "Hartree" and "Fock" diagrams with the replacement of the bare interaction by the $T$-matrix

$$
\begin{aligned}
& \Sigma_{12, \sigma}^{(T H)}(i \omega)=\frac{1}{\beta} \sum_{\Omega} \sum_{3 \sigma^{\prime}}\left\langle 13\left|T^{\sigma \sigma^{\prime}}(i \Omega)\right| 23\right\rangle G_{3}^{\sigma^{\prime}}(i \Omega-i \omega) \\
& \Sigma_{12, \sigma}^{(T F)}(i \omega)=-\frac{1}{\beta} \sum_{\Omega} \sum_{3}\left\langle 13\left|T^{\sigma \sigma}(i \Omega)\right| 32\right\rangle G_{3}^{\sigma}(i \Omega-i \omega) .
\end{aligned}
$$

Now we rewrite the effective Hamiltonian (15) with the replacement $\langle 12|v| 34\rangle$ by $\left\langle 12\left|T^{\sigma \sigma^{\prime}}\right| 34\right\rangle$ in $H_{U}$. To consider the correlation effects described due to P-H channel we have to separate density $(d)$ and magnetic $(m)$ channels as in Ref.[21]

$$
\begin{aligned}
d_{12} & =\frac{1}{\sqrt{2}}\left(c_{1 \uparrow}^{+} c_{2 \uparrow}+c_{1 \downarrow}^{+} c_{2 \downarrow}\right) \\
m_{12}^{0} & =\frac{1}{\sqrt{2}}\left(c_{1 \uparrow}^{+} c_{2 \uparrow}-c_{1 \downarrow}^{+} c_{2 \downarrow}\right) \\
m_{12}^{+} & =c_{1 \uparrow}^{+} c_{2 \downarrow} \\
m_{12}^{-} & =c_{1 \downarrow}^{+} c_{2 \uparrow} .
\end{aligned}
$$

Then the interaction Hamiltonian can be rewritten in the following matrix form

$$
H_{U}=\frac{1}{2} \operatorname{Tr}\left(D^{+} * V^{\|} * D+m^{+} * V_{m}^{\perp} * m^{-}+m^{-} * V_{m}^{\perp} * m^{+}\right),
$$

where $*$ means the matrix multiplication with respect to the pairs of orbital indices, e.g.

$$
\left(V_{m}^{\perp} * m^{+}\right)_{11^{\prime}}=\sum_{34}\left(V_{m}^{\perp}\right)_{11^{\prime}, 22^{\prime}} m_{22^{\prime}}^{+} .
$$

The supervector $\mathrm{D}$ is defined as

$$
D=\left(d, m^{0}\right), D^{+}=\left(\begin{array}{c}
d^{+} \\
m_{0}^{+}
\end{array}\right),
$$

and the effective interactions have the following form:

$$
\begin{aligned}
& \left(V_{m}^{\perp}\right)_{11^{\prime}, 22^{\prime}}=-\left\langle 12\left|T^{\uparrow \downarrow}\right| 2^{\prime} 1^{\prime}\right\rangle \\
& V^{\|}=\left(\begin{array}{cc}
V^{d d} & V^{d m} \\
V^{m d} & V^{d d}
\end{array}\right)
\end{aligned}
$$




$$
\begin{gathered}
V_{11^{\prime}, 22^{\prime}}^{d d}=\frac{1}{2} \sum_{\sigma \sigma^{\prime}}\left\langle 12\left|T^{\sigma \sigma^{\prime}}\right| 1^{\prime} 2^{\prime}\right\rangle-\frac{1}{2} \sum_{\sigma}\left\langle 12\left|T^{\sigma \sigma}\right| 2^{\prime} 1^{\prime}\right\rangle \\
V_{11^{\prime}, 22^{\prime}}^{m m}=\frac{1}{2} \sum_{\sigma \sigma^{\prime}} \sigma \sigma^{\prime}\left\langle 12\left|T^{\sigma \sigma^{\prime}}\right| 1^{\prime} 2^{\prime}\right\rangle-\frac{1}{2} \sum_{\sigma}\left\langle 12\left|T^{\sigma \sigma}\right| 2^{\prime} 1^{\prime}\right\rangle \\
V_{11^{\prime}, 22^{\prime}}^{d m}=V_{22^{\prime}, 11^{\prime}}^{m d}= \\
\frac{1}{2}\left[\left\langle 12\left|T^{\uparrow \uparrow}\right| 1^{\prime} 2^{\prime}\right\rangle-\left\langle 12\left|T^{\downarrow \downarrow}\right| 1^{\prime} 2^{\prime}\right\rangle-\left\langle 12\left|T^{\uparrow \downarrow}\right| 1^{\prime} 2^{\prime}\right\rangle\right. \\
\left.+\left\langle 12\left|T^{\downarrow \uparrow}\right| 1^{\prime} 2^{\prime}\right\rangle-\left\langle 12\left|T^{\uparrow \uparrow}\right| 2^{\prime} 1^{\prime}\right\rangle+\left\langle 12\left|T^{\downarrow \downarrow}\right| 2^{\prime} 1^{\prime}\right\rangle\right] .
\end{gathered}
$$

To calculate the particle-hole (P-H) contribution to the electron self-energy we first have to write the expressions for the generalized susceptibilities, both transverse $\chi^{\perp}$ and longitudinal $\chi^{\|}$. One has

$$
\chi^{+-}(i \omega)=\left[1+V_{m}^{\perp} * \Gamma^{\uparrow \downarrow}(i \omega)\right]^{-1} * \Gamma^{\uparrow \downarrow}(i \omega),
$$

where

$$
\Gamma_{12,34}^{\sigma \sigma^{\prime}}(\tau)=-G_{2}^{\sigma}(\tau) G_{1}^{\sigma^{\prime}}(-\tau) \delta_{23} \delta_{14}
$$

is an "empty loop" susceptibility and $\Gamma(i \omega)$ is its Fourier transform, $\tau$ is the imaginary time. The corresponding longitudinal susceptibility matrix has a more complicated form:

$$
\chi^{\|}(i \omega)=\left[1+V^{\|} * \chi_{0}^{\|}(i \omega)\right]^{-1} * \chi_{0}^{\|}(i \omega),
$$

and the matrix of the bare longitudinal susceptibility is

$$
\chi_{0}^{\|}=\frac{1}{2}\left(\begin{array}{ll}
\Gamma^{\uparrow \uparrow}+\Gamma^{\downarrow \downarrow} & \Gamma^{\uparrow \uparrow}-\Gamma^{\downarrow \downarrow} \\
\Gamma^{\uparrow \uparrow}-\Gamma^{\downarrow \downarrow} & \Gamma^{\uparrow \uparrow}+\Gamma^{\downarrow \downarrow}
\end{array}\right),
$$

in the $d d-, d m^{0}-, m^{0} d-$, and $m^{0} m^{0}$ - channels $\left(d, m^{0}=1,2\right.$ in the supermatrix indices). An important feature of these equations is the coupling of longitudinal magnetic fluctuations and of density fluctuations. It is not present in the one-band Hubbard model due to the absence of the interaction of electrons with parallel spins. For this case Eqs. (22) and (24) coincide with the well-known result of Izuyama et. al. [29].

Now we can write the particle-hole contribution to the self-energy. Similar to Ref.[22] one has

$$
\Sigma_{12, \sigma}^{(p h)}(\tau)=\sum_{34, \sigma^{\prime}} W_{13,42}^{\sigma \sigma^{\prime}}(\tau) G_{34}^{\sigma^{\prime}}(\tau)
$$

with the P-H fluctuation potential matrix: 


$$
W^{\sigma \sigma^{\prime}}(i \omega)=\left[\begin{array}{ll}
W^{\uparrow \uparrow}(i \omega) & W^{\perp}(i \omega) \\
W^{\perp}(i \omega) & W^{\downarrow \downarrow}(i \omega)
\end{array}\right],
$$

were the spin-dependent effective potentials are defined as

$$
\begin{aligned}
W^{\uparrow \uparrow} & =\frac{1}{2} V^{\|} *\left[\chi^{\|}-\chi_{0}^{\|}\right] * V^{\|} \\
W^{\downarrow \downarrow} & =\frac{1}{2} V^{\|} *\left[\tilde{\chi}^{\|}-\widetilde{\chi}_{0}^{\|}\right] * V^{\|} \\
W^{\uparrow \downarrow} & =V_{m}^{\perp} *\left[\chi^{+-}-\chi_{0}^{+-}\right] * V_{m}^{\perp} \\
W^{\downarrow \uparrow} & =V_{m}^{\perp} *\left[\chi^{-+}-\chi_{0}^{-+}\right] * V_{m}^{\perp} .
\end{aligned}
$$

Here $\tilde{\chi}^{\|}, \tilde{\chi}_{0}^{\|}$differ from $\chi^{\|}, \chi_{0}^{\|}$by the replacement of $\Gamma^{\uparrow \uparrow} \Leftrightarrow \Gamma^{\downarrow \downarrow}$ in Eq.(25). We have subtracted the second-order contributions since they have already been taken into account in Eq.(18).

Our final expression for the self energy is

$$
\Sigma=\Sigma^{(T H)}+\Sigma^{(T F)}+\Sigma^{(P H)} .
$$

This formulation takes into account accurately spin-polaron effects because of the interaction with magnetic fluctuations [30, 31], the energy dependence of the $T$-matrix which is important for describing the satellite effects in $\mathrm{Ni}$ [32], contains exact second-order terms in $v$ and is rigorous (because of the first term) for almost filled or almost empty bands.

Since the LSDA Green's function already contains the average electronelectron interaction, in Eqs. (18) and (26) the static part of the self-energy $\Sigma^{\sigma}(0)$ is not included, i.e. we have

$$
\tilde{\Sigma}^{\sigma}(i \omega)=\Sigma^{\sigma}(i \omega)-\Sigma^{\sigma}(0)
$$

\section{Results and discussion}

The matrix elements of $v$ appearing in Eq.(16) can be calculated in terms of two parameters - the averaged screened Coulomb interaction $U$ and exchange interaction $J$ [23]. The screening of the exchange interaction is usually small and the value of $J$ can be calculated directly. Moreover numeric calculations show that the value of $J$ for all $3 d$ elements is practically the same and approximately equal to $0.9 \mathrm{eV}$. This value has been adopted for all our calculations presented here. At the same time direct Coulomb interaction undergoes substantional screening and one has to be extremely careful making the choice for this parameter. There are some prescription how one can get it 

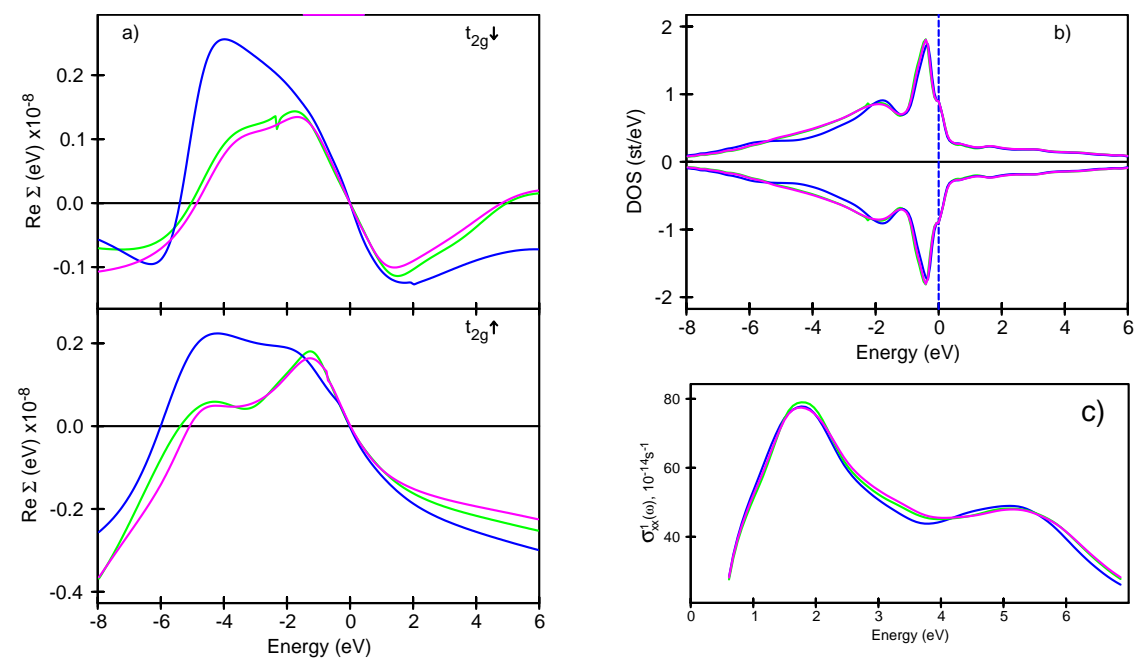

Figure 1. The self-energy (a) of Fe for three different temperatures and corresponding densities of states (b) and optical conductivities spectra (c). Full, dashed and dotted lines correspond to $T=125 K, T=300 K$ and $T=900 K$, respectively.

within constraint LDA calculation [2]. However, results obtained in this way depend noticeably on the choice of the basis functions, way of accounting for hybridization etc. Nevertheless the order of magnitude coming out from various approaches is the same giving the value of $U$ in the range $1-4 \mathrm{eV}$. In the present paper we are discussing the influence of the choice of $U$ on the calculated optical spectra.

Another parameter entering SPTF equations is temperature. For a moment we are more interested in the low temperature properties while computationally the higher the tempreture is, less computationally demanding are the calculations. This is why we decided first to consider the dependence of the selfenergy on the temperature.

In Fig. 1 we show the self-energy obtained for Fe for three different temperatures as well as corresponding densities of states and optical conductivities spectra. One can see that despite the differences in $\Sigma$ are quite noticeable this leads only to moderate changes in the density of states and does not affect the optical conductivity.

Much more important for the results is the parameter $U$. Fig. 2 shows as an example the real part $t_{2 g}$ component of $\Sigma$ for $T=300 K$ in Fe for various values of $U$. Despite the overall shape of the curve is practically the same the magnitude of the self-energy increasing with increase of $U$ as it is expected from the analytical expressions. This change in self-energy leads to corresponding changes in the densities of states especially noticeable for the 

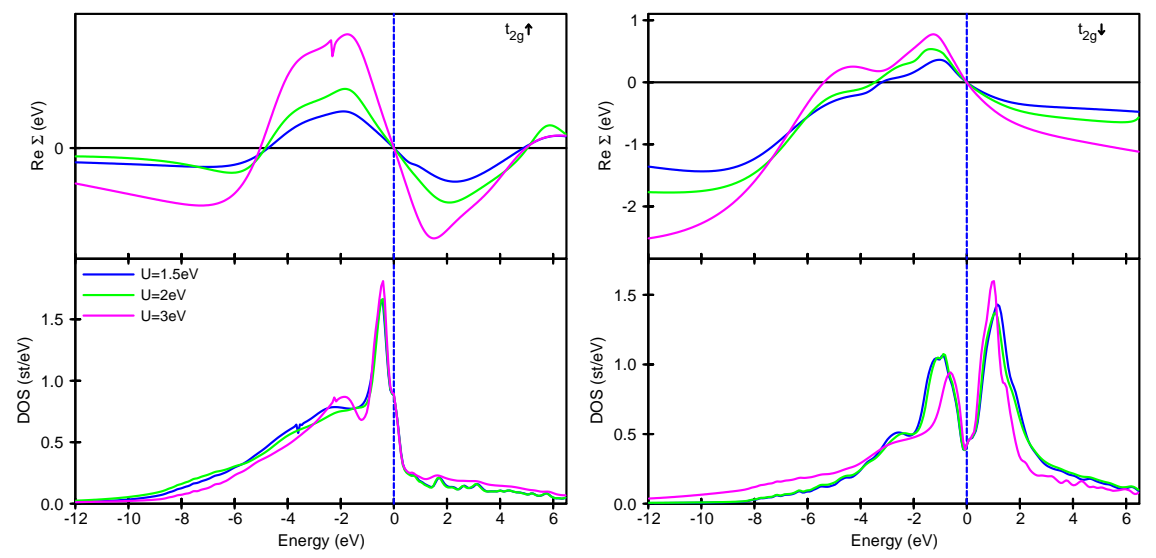

Figure 2. The real part of $t_{2 g}$ component of $\Sigma$ for $T=300 K$ in Fe for various values of $U$; left: spin-up, right: spin-down.

minority spin subband. The influence of the choice of $U$ on the optical properties is even more pronounced (see Fig. 3). The low energy peak in the diagonal part of the optical conductivity shifts to the lower energies reaching the experimental position already for $U=1.5 \mathrm{eV}$. In the high energy part of the spectra large values of $U$ lead to a structure around $5 \mathrm{eV}$ not seen in experiment.

Again, the value $U=1.5 \mathrm{eV}$ gives also the best description for the shape of the experimental curve. (Note, that the experimental results for $\sigma_{x x}^{1}(\omega)$ are multiplied by a factor of 1.7 to make the comparison more obvious.) A rather different situation occurs for the off-diagonal part of the optical conductivity.
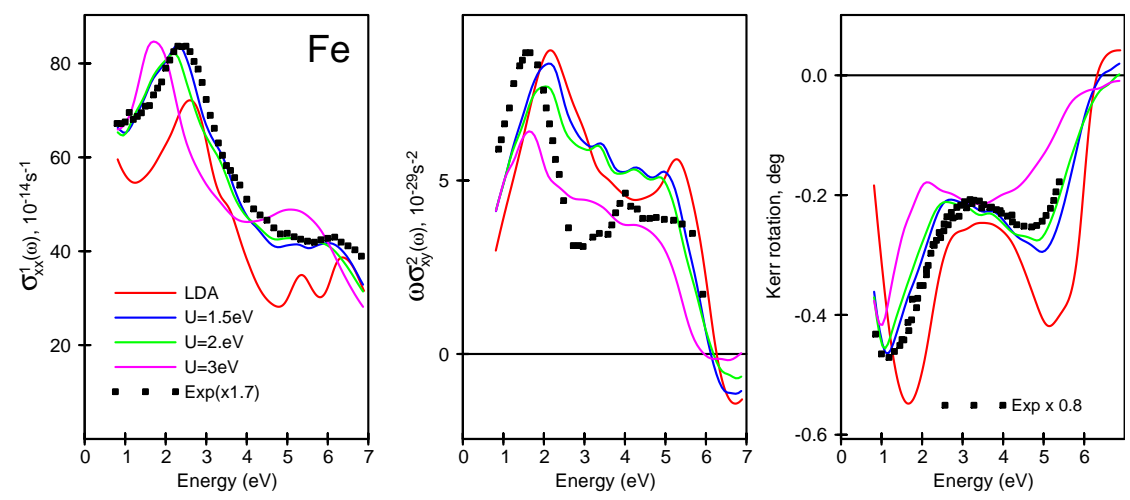

Figure 3. Optical conductivity (left: diagonal; middle: off-diagonal) and polar Kerr rotation (right) spectra in comparison with the experimental data of Fe. Experimental data for conductivity are taken from Ref. [33, 34]; Kerr rotation spectra - from Ref. [35] 

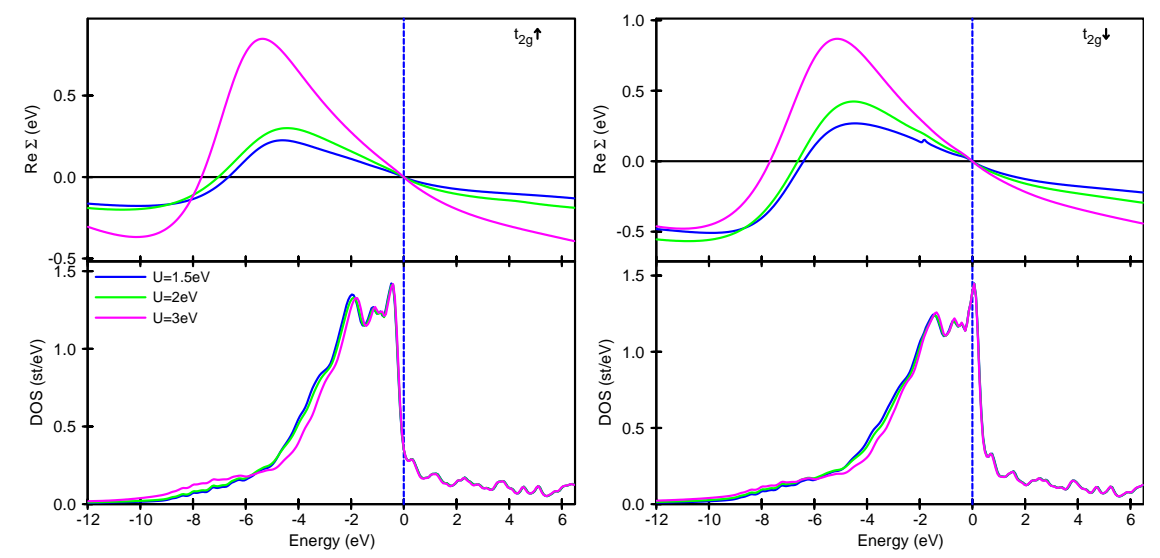

Figure 4. The real part of $t_{2 g}$ self-energy for $U=1.5,2,3 \mathrm{eV}$ and corresponding DOS plots for Ni; left: spin-up, right: spin-down.

The low energy peak can be brought to the proper position only with $U=4 \mathrm{eV}$, at the same time the shape of the theoretical curves above $2.5 \mathrm{eV}$ has a rather different structure in comparison with the experimental one, only crossing the zero axis at the same energy. However, a direct comparison of calculated $\sigma_{x y}$ data with experimental ones may be somewhat misleading as experimentally this quantity cannot be measured directly and is usually obtained from ellipsometric measurements and measurements of the Kerr rotation spectra. Thus in the left panel of the Fig. 3 we show our results for the calculated polar Kerr rotation spectra in comparison with experimental data. As one can see again the DMFT calculation with $U=1.5 \mathrm{eV}$ describes the experimental data in a rather satisfactory way.

If for Fe LSDA calculations already give a reasonable description of the optical properties and the many-body correlation effects, which improves only minor details, the situation in $\mathrm{Ni}$ is quite different. It is well-known that LSDA fails to describe the bandwidth for $\mathrm{Ni}$, causing problems in the theoretical interpretation of all the spectroscopic experiments such as photoemission, $\mathrm{x}$-ray emission, optics, etc. The main reason for this is the underestimation of electron-electron correlations which appear to be relatively strong in this metal. Again, as in the case of Fe, we carried out calculations with different values of $U$ to find the best description of the spectral properties of $\mathrm{Ni}$. In Fig. 4 we show the real part of the $t_{2 g}$ self-energy for $U=1.5,2,3 \mathrm{eV}$ as well as corresponding DOS plots.

Despite the changes in the amplitude of the self-energy are important, all self-energies lead to rather small changes in the density of states, narrowing somewhat the bandwidth only and developing a low energy tail. Nevertheless 

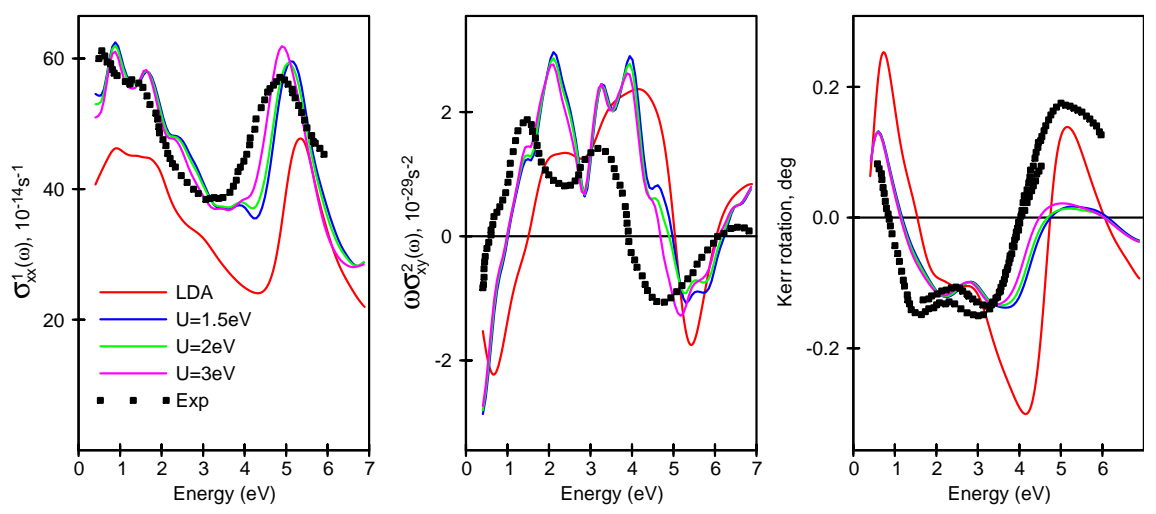

Figure 5. Optical conductivity and polar Kerr rotation spectra in comparison with the experimental data of Ni. Experimental data for conductivity are taken from Ref. [36, 37]. Kerr rotation spectra - from Ref. [35]

the diagonal part of the conductivity which reflects the convolution of the occupied and unoccupied states is much more affected by the choice of $U$. The main change can be seen in the position of high energy peak which is placed by LDA about $1 \mathrm{eV}$ higher in comparison with experiment. Accounting for the correlation effects shifts this maximum bringing it to the proper position for $U=3 \mathrm{eV}$. The low energy part of the spectra does not reflect too much influence of the $U$ parameter and deviates just slightly from the experimental curve. For the off-diagonal part of the conductivity an improvement as compared to LDA is not so pronounced as for the diagonal one, though the spectra getting closer to experiment. It is worth to note that the actual value of $U$ doesn't change the calculated spectrum of $\omega \sigma_{x y}^{2}(\omega)$. But again, as mentioned in the case of $\mathrm{Fe}$ it is worth to compare calculations with directly measured Kerr rotation spectra presented in Fig. 5. As one can see, the improvement compared to LSDA results is substantional but our results are still far from experiment concerning the peak position both in the infrared and visible parts of the spectra. This disagreement is apparently coming from the approximation that has been made and is much more pronounced in the off-diagonal part of conductivity as it is more sensitive to the details of the electronic structure being the result of complex interplay of exchange splitting and spin-orbit coupling.

It is still unclear whether the mentioned problems are coming from the single-site approximation for the self-energy (DMFT) itself or whether they are reflecting the limitations of the simplified FLEX method of solving the impurity many-body problem. To find out an answer more elaborated solvers like QMC have to be used. 


\section{Conclusion and outlook}

In the present paper we show a way to account for the particle-particle correlations in the theoretical description of optical and magneto-optical properties of the ferromagnetic $3 d$ metals. We show that the dynamical correlations play an important role even in weakly correlated materials like Fe and can substantially change the shape of the spectra for moderately correlated Ni. Even a rather simple way of accounting for dynamic correlation allows to improve theoretical results substantionally though not giving the perfect agreement with experiment.

Thus to go further one has to use more elaborated technique to obtain the self-energy both within DMFT and beyond (for example, new DMFT+GW approximation). Work along this line is in progress.

\section{References}

[1] P. Hohenberg and W. Kohn, Phys. Rev., 136, B864 (1964).

[2] R. O. Jones and O. Gunnarsson, Rev. Mod. Phys. 61, 689 (1989).

[3] W. Kohn and P. Vashishta, in: Theory of the Inhomogeneous Electron Gas, Ed. by S. Lundqvist and N. H. March, p. 79. Plenum, New York (1983).

[4] C.-O. Almbladh and U. von Barth, in: Density Functional Methods in Physics, Ed. by R. M. Dreizler and J. da Providencia, p. 209. Plenum, New York (1985).

[5] G. Borstel, Appl. Phys. A 38, 193 (1985).

[6] L. Hedin, Phys. Rev. 139, 796 (1965).

[7] F. Aryasetiawan, Phys. Rev. B 46, 13051 (1992).

[8] M. Rohlfing, P. Kruger, and J. Pollmann, Phys. Rev. B 48, 17791 (1993).

[9] F. Aryasetiawan and O. Gunnarsson, Phys. Rev. Lett. 74, 3221 (1995).

[10] Anisimov V. I., Zaanen J. and Andersen O. K., Phys. Rev. B, 44 (1991) 943.

[11] A.N. Yaresko, P.M. Oppeneer, A.Ya. Perlov, V.N. Antonov, T. Kraft, and H. Eschrig, Europhys. Lett. 36, 551, (1996).

[12] V. N. Antonov, V. P. Antropov, B. N. Harmon, A. N. Yaresko, and A. Ya. Perlov Phys. Rev. B 59, 14552,(1999).

[13] A. Georges, G. Kotliar, W. Krauth, and M. Rozenberg, Rev. Mod. Phys. 68, 13 (1996).

[14] P. W. Anderson, in Moment formation in solids, edited by W. J. L. Buyers, Plenum Press, New York and London, 1984, p. 313.

[15] A. Georges, G. Kotliar, Phys. Rev. B 45, 6479 (1992).

[16] H. Keiter, J. C. Kimball, Phys. Rev. Lett., 25, 672 (1970)

[17] N. E. Bickers, D. L. Cox, J. W. Wilkins, Phys. Rev. B 36, 2036 (1987)

[18] J. E. Hirsch, R. M. Fye, Phys. Rev. Lett. 56, 2521 (1986); M. Jarrel, Phys. Rev. Lett. 69, 168 (1992); M. Rozenberg, X. Y. Zhang, G. Kotliar, Phys. Rev. Lett. 69, 1236 (1992); A. Georges, W. Krauth, Phys. Rev. Lett. 69, 1240 (1992).

[19] M. Caffarel, W. Krauth, Phys. Rev. Lett. 72, 1545 (1994)

[20] R. Bulla, Adv. Sol. State Phys. 46, 169 (2000) 
[21] N. E. Bickers, D. J. Scalapino, Ann. Phys. (N.Y.) 193, 206 (1989)

[22] M. I. Katsnelson and A. I. Lichtenstein, J. Phys.: Condens. Matter 11, 1037 (1999)

[23] M. I. Katsnelson and A. I. Lichtenstein, Eur. Phys. J. B 30, 9 (2002)

[24] T. Huhne, H. Ebert, Phys. Rev. B 60, 12982 (1999).

[25] V. N. Antonov, A. N. Yaresko, A. Ya. Perlov, V. V. Nemoshkalenko, Low Temperature Physics 25, 387 (1999)

[26] R. Kubo, J. Phys. Soc. Jpn. 71, 585 (1958)

[27] O. K. Andersen, Phys. Rev. B 12, 3060 (1975).

[28] A. Perlov, S. Chadov, H. Ebert, Phys. Rev. B 68, 245112 (2003).

[29] T. Izuyama, D. Kim, and R. Kubo, J. Phys. Soc. Japan 18, 1025 (1963).

[30] Yu. Irkhin and M. I. Katsnelson, J. Phys. : Condens. Matter 2, 7151 (1990).

[31] V. Yu. Irkhin and M. I. Katsnelson, Physics - Uspekhi 37,659 (1994).

[32] A. Liebsch, Phys. Rev. B 23, 5203 (1981).

[33] H.T. Yolken, J. Kruger, J. Opt. Soc. Am. 55, 842 (1965)

[34] G. S. Krichnik, V. A. Artem'ev, Sov. Phys. J.E.T.P. 26, 1080 (1968)

[35] P.G. van Engen, Ph.D. thesis, Tech. Univ. of Delft (1983), S. Visnovsky et al., J. Magn. Magn. Materials 127, 135 (1993)

[36] P. B. Johnson, R. W. Christy, Phys. Rev. B 9, 5056 (1974)

[37] J. L. Erskine, Physica B 89, 83 (1977) 